\title{
Brucellosis Control
}

Brucellosis, both a human health and an agricultural economic problem, was the subject of a special session at Pennsylvania's Second Annual Health Conference held in 1953. The consensus of the five speakers at the session was that, with the epidemiological aspects and the preventive and control measures well established, brucellosis can be eradicated, if a strong policy is developed to apply the recommended procedures. Dr. Milo reported on Pennsylvania's progress in eliminating the disease in livestock, and Dr. Dean spoke on public health control activities. Dr. Dean's paper is presented in full; condensations of the other papers follow.

\section{The Public Health Approach}

\author{
BY JOSEPH O. DEAN, M.D.
}

$\mathrm{B}$ RUCELLOSIS is the most prevalent disease of animals transmissible to man, and therefore public health officials are concerned with its control. On the public health side are such control measures as education, improved reporting of cases and diagnostic criteria, and the development of uniform laboratory aids essential to the diagnosis of human brucellosis.

But public health officials see the problem as one they can successfully cope with only as

Dr. Dean was associate chief of the Bureau of State Services, Public Health Service, at the time of the Pennsylvania conference. He is now an assistant to the medical director of the Bureau of Indian Affairs, Department of the Interior. the veterinary profession and livestock sanitary officials succeed in eradicating the disease in domestic animals. Under the present limits of knowledge, the prevention of human brucellosis is dependent upon preventing exposure to infected animals and the infectious tissue or products of such animals. We have no other direct preventive measures, such as vaccination, for human brucellosis.

The human illness resulting from infection through the livestock reservoir, while important whether measured in suffering or direct economic loss, is not the whole consideration. Certainly, in the long run, food supply has to be considered as well.

Now of lesser concern, the problem of nutrition will grow as the population of the United States expands beyond the capacity of agriculture to supply the food it demands. Maximum 
production per animal unit becomes progressively more important to us and compels in effect a fusion of the agricultural economic and public health viewpoints. Dr. B. T. Simms of the United States Department of Agriculture has pointed out that history reveals no record of a well-fed nation which allowed animal diseases to go uncontrolled. In the past 40 years, our per capita meat consumption has dropped about 10 percent, and since 1930 the per capita milk consumption has also dropped.

In the United States, the policy of attempting to eradicate important livestock diseases has been adopted. The prevailing philosophy has been to live without rather than with many of the diseases which affect domestic animals and especially those transmissible to man.

\section{National Plan}

The problem of eradicating brucellosis is very difficult and has been hindered by a good deal of disagreement in the past. There is sufficient agreement now among livestock sanitary officials on the basic procedures necessary in an eradication program to command the support of public health workers. Effective support will require an acquaintance with the national plan of the United States Livestock Sanitary Association and with the recommendations of the National Research Council's Committee on Public Health Aspects of Brucellosis in its report on the control and eradication of brucellosis in animals.

The national plan and the committee's report make apparent the necessity for regulations that govern the movement of livestock across national, State, and farm boundaries so as to minimize the risk of introducing or extending infection. In our agricultural and livestock economy, the intermingling of animals from different herds is one of the most serious obstacles to the eradication of disease. With the steady growth of livestock traffic through trucking and other transport and through sales barns, the need for livestock regulation also grows.

There are a number of things public health personnel can and should do to aid in brucellosis eradication. We need to have at least a speaking acquaintance with the facts and proce- dures upon which present efforts are based if we are to support those more directly engaged in the work.

We can help foster a wider appreciation of the disadvantages, both economic and healthwise, of infection in farm and range animals. Certainly the occupational hazard of brucellosis needs highlighting. The dependence of the packing plant workers upon the stockman for protection and the responsibility of the stockmen to the packing plant workers are insufficiently understood. Public health agencies can intensify their educational and information efforts to improve the understanding of the public, particularly workers in slaughter houses, packing houses, and butcher shops, as to the nature of the disease and the feasibility of its control and the costs of living with brucellosis compared to banishing it.

As control progresses, closer liaison with veterinary and livestock sanitary officials takes on an added importance. Human cases will be traced to the common or individual source. That information placed in the hands of those engaged in the eradication of brucellosis in animals will serve as a check on where infection has been missed in the testing program. Likewise, by making available to public health officials information on the location of infected herds, veterinarians can assist the health department in tracing sources of human infection.

\section{New Milk Ordinance}

Milk ordinances have had an important influence upon the dairy industry. Certainly, public health policy has been a strong factor in motivating the production of milk from diseasefree animals.

The fact that about 90 percent of the market milk in the United States is pasteurized undoubtedly is related to the fact that only about one-fourth of human brucellosis is traceable to consumption of milk or dairy products. The remainder is traceable largely to direct contact with infected animals or carcasses. Our goal, however, must be pasteurization of all market milk. This would further reduce the number of human cases. But more than pasteurization is required. Because mechanical and human failures can occasionally defeat the most con- 
scientious sanitation program, milk from uninfected herds must be the aim.

Under the new 1953 milk ordinance and code, recommended by the Public Health Service, all milk producers in States and communities adopting the ordinance must be operating under plans A or B within 3 years of its adoption. Plan A specifies testing of infected herds, immediate slaughter of all adult reactors, thorough cleaning and disinfection of barns and buildings in which reactors have been kept, and retesting at intervals of at least 30 days until the disease is eradicated. Plan $B$ requires testing of all adult animals, but permits temporary retention of reactors in the herd until they can be disposed of by slaughter without excessive financial loss to the owner. Calf vaccination is optional in both plans.

In either plan, all additions to the herd, except calves born into the herd or vaccinated, have to be free of brucellosis as determined by a negative test given not more than 30 days prior to such addition. Calf vaccination is permitted in herds required to be free of brucellosis if the calves have been vaccinated between 4 and 8 months of age with a vaccine approved by the United States Department of Agriculture and carry a blood agglutination titer no higher than incomplete in a 1:100 dilution, which subsequently stabilizes or drops below this level.

Ultimately, the standard ordinance will specify that all milk-producing herds be under plan A. Health officers wishing to assist the State and Federal livestock disease control officials in developing brucellosis-free certified herds and areas should file a request for cooperative testing with the State veterinarians. The provisions of the new ordinance and code are compatible with, and follow closely, the recommendations of the United States Livestock Sanitary Association and the program approved by the United States Department of Agriculture, and I am certain will in every sense aid the program.

The 1939 ordinance had no requirement for brucellosis control with respect to milk intended for pasteurization. It was a serious shortcoming. However, the adoption of the code by 1,565 municipalities and by 405 counties and its use in framing laws and regulations in 34
States give a fair insight into the effect we may expect the new ordinance to have on brucellosis eradication among dairy cattle.

As pointed out, control of human brucellosis is primarily achieved by informing the public and selected occupational groups about the nature of the disease, mode of transmission, and danger of infected milk and carcasses, and by attacking the animal reservoirs. The latter calls for discovery of infection among livestock by agglutination reaction and the elimination of reactors from herds by segregation or slaughter, vaccination of calves and pasteurization of milk.

\section{Recognition and Diagnosis}

However, much remains for public health officials to do about individuals who acquire the disease.

To begin with, we need much better recognition of the disease. Recognition is difficult because most human brucellosis appears as a sporadic disease and simulates many other illnesses in its symptomatology. Recognition has been confused by overemphasis of the chronic aspects of the disease and misplaced trust in the dermal sensitivity test. Only a small proportion of cases represent chronic brucellosis, lasting over a period of months and years. The dermal sensitivity test is generally regarded as being of no aid in the diagnosis of a suspected case and as having no place in clinical medicine.

A definite diagnosis can only be made with the aid of laboratory procedures, namely, cultural studies and the agglutination test. Cultural studies should be made on every patient suspected of having brucellosis. Increasing use is being made of sternal bone marrow when culture of venous blood has been unsuccessful.

With the increased tendency to use antibiotics for febrile conditions of unknown etiology, the agglutination test is being depended on more and more for the diagnosis of brucellosis.

The reliability of the test depends on the antigen used. There is a wide variability among antigens and test procedure in current use, with accompanying variability in the specificity and reliability of results.

The success of the program for the control 
and eradication of brucellosis in domestic animals is due largely to the use of standardized antigen and a standardized test procedure. A number of public health laboratories have found the antigen prepared by the United States Department of Agriculture very satisfactory, and the extension of its use to all clinical and public health laboratories has recently been recommended by the Association of State and Territorial Health Officers.

It has been said the clinician should not depend on a so-called diagnostic titer. In general, but by no means surely, the higher the titer the more likely it represents infection. In experiments on human volunteers, individuals fed heat-killed Brucella abortus organisms in quantities that might be found in pasteurized milk from infected cows developed agglutinins against this organism in fairly high titer. In several instances, agglutination in a 1: 640 dilution of the serum was obtained.

However, the great majority of culturally proved cases are associated with a titer of $1: 100$ and above. Often agglutinins may be demonstrated when the serum is diluted 10 to 20 thousand times. In groups repeatedly exposed to Brucella, such as farmers, meat packing plant employees, and veterimarians, even the higher titers lose diagnostic significance because many of these persons have titers of $1: 320$ or higher without being ill. Rise of agglutination titers in persons who have recently had a skin test likewise has little, if any, diagnostic significance. Apparently, for practical purposes, therapy with antibiotics does not interfere with the diagnostic value of the agglutination test.

Better recognition of cases, then, necessitates more rigid diagnostic criteria and reliance on laboratory procedure for confirmation of diagnosis, the preference being for isolation of the infecting organism. Use of a standard reliable antigen and test procedure subjected to periodic evaluation of results will substantially enhance the value of the agglutination test and the identification of cases. The skin test-dermal sensitivity test-should be abandoned.

\section{Case Reporting}

Finally, reporting of cases to the health departments is essential. Given accurate diag- nosis and adequate recognition of cases by the clinician, the case still must be traced to its source and the source eliminated, if a complete job is to be done. This epidemiological tracking down of sources cannot be done feasibly in the absence of good reporting.

Over the last quarter century the number of cases of reported human brucellosis rose from less than 100 to more than 6,300 in 1947 . Since 1947 , there has been a steady decrease, with only 2,537 cases reported nationally in 1952. How much of this decline represents more accurate diagnosis or how much of it reflects an actual decrease in the incidence of human brucellosis, we do not know. We are confident, of course, on the basis of epidemiological studies made by various investigators that recognition and reporting of cases is very incomplete. At the same time, in known instances the reported cases in a State have dropped inside the areas or counties where brucellosis eradication work in animals was progressing, and concomitantly an actual increase occurred in the remainder of the State.

In the absence of other obvious, practical incentives, responsibility for eliminating brucellosis from animal herds rests for the time being almost entirely upon public health policy.

\section{Human Brucellosis}

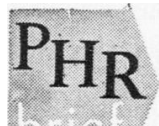

Brucellosis is a disease of animals, mainly domestic animals, of worldwide distribution. Man acquires the disease usually by direct contact with infected animals, their excretions, secretions, or tissues, or by ingesting unpasteurized mill or other dairy products derived from infected animals. While Brucella abortus is found mainly

By Norman B. McCullough, M.D., chief, Laboratory of Clinical Investigation, National Microbiological Institute, National Institutes of Health, Public Health Service. 
in cattle, Brucella suis in swine, and Brucella melitensis in goats and sheep, any of the three species may occur in any of these animals. In the United States, cattle and hogs are the chief reservoirs. Thus, most of our human infections are due to $B r$. abortus or $B r$. suis. In this country, Br.melitensis is present mostly in swine and constitutes an additional public health hazard. In public health importance, brucellosis now ranks as the leading disease of animals transmissible to man.

With the pasteurization of most of the commercially distributed milk, this avenue of transmission is becoming less important. In epidemiological studies conducted during the last few years, approximately 75 percent of the human cases have been shown to be due to direct contact with infected animals or their tissues. Brucellosis is an occupational disease, mainly affecting those groups intimately in contact with infected animals or their carcasses-the cattle and swine raiser, dairyman, packing plant worker, meat handler, and veterinarian.

Clinically, human brucellosis, also known as undulant fever or malta fever, can present a varied picture. The acute case is characterized by high fever, extreme weakness, and excessive sweating. The onset may be abrupt or gradual, and the disease may last for a few days or weeks, without subsequent recurrence, or there may be one or more recurrences after the initial episode. In other cases, the disease may last for months or years with low-grade fever and multiple symptoms such as weakness, lassitude, and generalized aching, with remission and recurrences. The organism may localize in any part of the body. The symptoms are then dependent on the site of localization. A great many cases are probably entirely subclinical and recognizable illness does not result.

With the advent of the antibiotics, treatment of human brucellosis is now available. The broad spectrum antibiotics by themselves suppress the disease, but the relapse rate is high. The most efficacious treatment makes use of a combination of drugs. Dihydrostreptomycin, aureomycin, and sulfadiazine (or triple sulfonamide) given concurrently is the therapy of choice. Treatment must be intensive and prolonged to reduce the risk of relapse. Bed rest and general therapy are important items in the treatment. Localized complications may require special therapy.

\section{Pathology and Transmission}

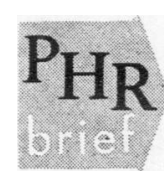

Brucella organisms may enter the body through the skin, the conjunctiva, and the digestive, genital, and respiratory tracts.

The digestive tract is believed the main portal of entry for cattle and swine. But for swine the genital tract is also important, because the boar is much more frequently infected than the bull, and discharges large numbers of Brucella with the semen during breeding.

In man, most infections are acquired through the skin from contact with infected fetuses, placentas, or other animal tissues. Brucella may enter through any wound or abrasion, but even the intact skin is no barrier against this organism.

Ticks, flies, bedbugs, and other ectoparasites, feeding on infected material, can spread the organisms through intestinal droplets deposited on the skin or conjunctiva. Dust particles, stirred up from infected litter, may be inhaled and lead to infection.

Establishment of Brucella in the tissues after it has penetrated one of the barriers depends on a number of factors: the pathogenicity of the three Brucella species; the number and virulence of the infecting organisms; natural or acquired resistance of the host; age of the host; and stage in the reproductive cycle of the host.

Some species, such as the rat, mouse, chicken, and hamster, are somewhat more resistant to Brucella infection than the cow, hog, guinea pig, rabbit, and man, and larger doses of $B$ rucella are needed to establish infection in the former group.

By I. Live, V.M.D., Ph.D., professor of microbiology, School of Veterinary Medicine, University of Pennsylvania. 


\section{Young Are Resistant}

The age of the individual seems to have a definite relationship to susceptibility to Brucella infection. Cattle are resistant up to breeding age. Under natural or artificial exposure such animals develop only a low agglutinin titer of short duration, and the infection is transient. According to various studies, children are more resistant to infection than adults. Swine of all ages are susceptible, but the effects of the disease are more pronounced in older animals than in the young. The increase in the susceptibility with sexual maturity may be due to the appearance of certain hormones at puberty, or it may be the result of the development of tissues which Brucella prefers for localization, such as the chorionic epithelium and the mammary gland.

Pregnancy is known to enhance the establishment of the infection. Fully mature nonpregnant cattle are generally less susceptible to permanent infection than pregnant cows. This fact and the knowledge that Brucella tends to localize in the pregnant uterus and through it infect the fetus lead to the conclusion that Brucella has predilection for embryonal tissues. The lymph nodes, joints, spleen, liver, and bone marrow also are frequently involved.

Histopathological studies have revealed that the organisms invade the tissue cells, thus leading an intracellular existence similar to that of filterable viruses. This is important in the treatment of brucellosis. The antibiotics and sulfonamides used cannot be expected to reach the organisms within the cells, and they affect mainly the Brucella in the body fluids. Thus, the intracellular organisms may survive a course of treatment, and eventually cause the recurrence of clinical symptoms. The intracellular parasitism may also be responsible for the chronic conditions, because the Brucella within the tissue cells is protected from the bactericidal action of the serum.

\section{Avenues of Elimination}

The most common and dangerous avenue of elimination of infected organisms is from the infected uterus at the time of abortion or parturition. Brucella may continue to be eliminated from the uterus exudate for several weeks or occasionally even longer.
In the pregnant animal, the infecting organisms invariably invade the cells of the maternal placenta (chorion). There is destruction of cells, accumulation of exudate between the maternal and fetal placenta, and adhesion between the two layers. If a large area of the chorionic epithelium is involved, the fetal circulation may be sufficiently interfered with to cause asphyxia and death of the fetus, and the animal aborts.

If the animal is infected late in pregnancy or has developed some resistance, the damage produced may not be severe enough to cause abortion. But the bacteria will be there and will be eliminated at parturition. In cattle, abortion usually occurs during the second half of pregnancy, but in swine abortion may occur at any stage of gestation, sometimes so early that it may pass unnoticed. The adhesions between the fetal and maternal placenta may result in a retained placenta which often has to be removed manually.

Another important mode of elimination of Brucella is through milk from an infected udder. It is estimated that the mammary gland is infected in the majority of cases of brucellosis in cows. Nursing calves which drink milk containing Brucella do not become permanently infected because young bovines are resistant. However, the organisms may pass intact through the digestive tract of the calves, thus spreading the infection to susceptible animals which may come in contact with the contaminated fecal matter.

\section{Viability}

After elimination from the animal body, the organisms remain alive for varying periods of time, depending upon the environment and the conditions under which they are kept. Brucella is readily destroyed by exposure to heat which is lethal to other nonspore-forming bacteria. In suspension, all three species of Brucella are killed in 20 minutes at $60^{\circ} \mathrm{C}$. Direct sunlight is lethal to the organisms within several hours. On the other hand, Brucella will survive for a long time when exposed to cold temperatures. Brucella suis has been recovered from an infected spleen kept at $-10^{\circ} \mathrm{F}$. for 30 days. Brucella abortus was alive in in- 
fected exudate from the bovine uterus 7 months after being stored in an ice chest. $B r$. abortus remained viable for 30 days in ice cream stored at $32^{\circ} \mathrm{F}$. and in butter kept at $46.5^{\circ} \mathrm{F}$., for 142 days.

Brucella appears sensitive to the action of an acid environment. It dies rather rapidly in milk kept at room temperature and survives longer at refrigerator temperatures.

In cheddar cheese produced from infected milk and permitted to ripen for 1 year no Brucella could be recovered.

\section{The Pennsylvania Program}

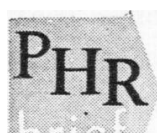

The bureau of animal industry of the Pennsylvania Department of Agriculture, in cooperation with the United States Department of Agriculture, has been waging a campaign against brucellosis in livestock in Pennsylvania since August 1934.

Federal indemnity covering reactors found as a result of the agglutination test for brucellosis was available to herd owners in Pennsylvania August 1, 1934, and State funds covering indemnities were available May 1, 1937. Since that time herd owners in this State have received more than $\$ 13$ million from the State and Federal governments. Results attained by this expenditure of public funds and the efforts of the veterinary profession and other cooperating agencies in combating this disease follow.

\section{County and Herd Tests}

As of July 1, 1953, 25 of the 67 counties in Pennsylvania were certified as free of brucellosis. Areas are certified when the incidence of the disease has been reduced to 1 percent or less

By H. A. Milo, V.M.D., director, bureau of animal industry of the Pennsylvania Department of Agriculture. and the percentage of infected herds does not exceed 5 percent. The last brucellosis test of the cattle population in these 25 counties showed that the percentage of infection had been reduced to 0.7 percent. In addition, 8 counties have been completely tested and every indication points to the fact that the next countywide test will certify these 8 counties. Twenty-three other counties have been partially tested.

On July 1, 1953, Pennsylvania had 75,404 herds, comprising 827,085 cattle, under supervision for brucellosis. This is approximately 50 percent of the total number of herds and cattle in the State.

To attain these results it was necessary to test 610,490 herds, comprising 6,216,590 cattle. This includes initial and retests of herds since August 1, 1934.

We also have 355 goat herds, comprising 2,904 goats, under supervision. Pennsylvania does not have a program at this time covering the testing of swine herds for brucellosis. However, owners may receive this service from their local veterinarian.

\section{Calf Vaccination}

Calf vaccination is considered a valuable adjunct to the brucellosis program, and, since January 1, 1950, the State has supplied Brucella abortus vaccine to practicing veterinarians for vaccinating calves, and it has also remunerated them for vaccination services.

During 1952, 136,000 calves were vaccinated in connection with the brucellosis program, and during 1953 a total of 700,000 calves were vaccinated.

Calves vaccinated between the ages of 6 and 8 months are subject to State and Federal indemnity if they give a positive reaction to the test when they are more than 30 months of age. Calves between 8 and 10 months of age may be vaccinated at the owner's request. However, they are not subject to State or Federal indemnity if they react positively after reaching the age of 30 months. If a calf over 30 months of age gives a suspicious titer to the test, the owner may present it for a retest. If the retest does not exceed a titer of over $1: 100$, or declines, the owner will not lose the status of a certified herd at the time of the last herd test. 
Calf vaccination has been most successful in that it gives the herd owner an opportunity of replacing older animals with heifers that have a certain amount of immunity when exposed to infection.

\section{Funds Reduced}

For the first time in the history of the brucellosis control and eradication program, Pennsylvania is in a position to keep up with the present schedule and to test areas that may qualify in the future. The expansion of the program will depend entirely upon State and Federal funds available for indemnity purposes.

Federal funds for indemnity purposes covering brucellosis reactors have been reduced considerably, and it has not been determined what portion of these funds will be allocated to Pennsylvania and what restrictions will be necessary in order to make these funds reach as far as possible and to use them where they will accomplish the greatest amount of good.

\section{Veterinarian's Viewpoint}

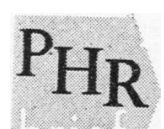

Veterinarians have found that regular testing of herds and elimination of infected animals, calf vaccination, and proper sanitation are all required to eradicate brucellosis. No one method suffices.

The test and slaughter method has been effective in reducing the incidence of brucellosis on an area basis in Pennsylvania when economic conditions have been favorable for the herd owners. In 1934, when brucellosis control was instituted on a national basis as part of a cattle reduction program, Federal and State funds became available for the payment of indemnities,

By C. J. Hollister, D.V.M., general veterinary practitioner, Montrose, $P$ a. for veterinary services, and for laboratory procedures.

Farmers cooperating received enough from indemnities and from slaughter salvage to replace condemned animals. Many herds were replaced several times during this period. But when prices for cattle and milk products became high commercial dairymen and beef producers were no longer keenly interested in disposing of infected animals. Problems began to arise for regulatory officials and the veterinarians conducting the test and slaughter program. Some owners refused to have their animals tested. Although brucellosis was being successfully controlled in thousands of herds and in many areas of Pennsylvania, it became obvious that it was not economically feasible nor practical to continue on a test and slaughter basis without some adjunct.

\section{Vaccination Results}

In 1941, Brucella abortus vaccine, strain 19, came into general use. We had, in addition to other methods of control, calf vaccination and adult vaccination with retention of positive animals.

Immunity conferred by Brucella abortus vaccine is not absolute nor permanent and can be broken down by continued exposure. But vaccine alone will go a long way toward preventing abortions and resultant sterility. When this clinical evidence of brucellosis has been controlled, it is difficult to convince the owners to test and eliminate the residual infection, which is a potential danger to both animals and man.

In untested areas where vaccine has been used to check the symptomatic course of the disease, owners are pleased with the results. When abortions stop the owner feels that brucellosis has been controlled and nothing further needs to be done. This, of course, is not true since after an abortion storm, the disease becomes chronic, a potential cause of fresh outbreaks.

The use of vaccine in calves in clean herds is not without its problems. Many veterinarians have seen calfhood-vaccinated purebred cattle penalized because they have not returned to a negative status by the time they are 30 months of age. Veterinarians sometimes won- 
der if they should continue "selling" calf vaccination in these herds, when they see a branded reacting animal bring 4 cents a pound less than an actinomycotic lumpy-jawed animal that was not branded.

There is another problem that needs appraisal. Since Brucella suis, the main cause of brucellosis in swine, and Brucella melitensis, the cause of brucellosis in goats, are pathogenic to cattle, the question is whether brucellosis can be eradicated in cattle without also eliminating the disease from these species. Indeed the complexity of the whole brucellosis problem is difficult for the average herd owner to understand.

\section{Accelerated Program}

Pennsylvania now has an accelerated program as a direct result of the determination of public health officials to eliminate brucellosis from cattle herds and thereby eliminate one of the causes of brucellosis in man.

A statewide brucellosis committee was formed in 1949 to facilitate eradication of the disease within Pennsylvania and to establish it as a "modified accredited brucellosis-free State." Its origin stemmed from a joint meeting of a group of agricultural representatives with representatives of the United States Department of Agriculture, the Pennsylvania Department of Agriculture, the Pennsylvania State College, and the Pennsylvania State Veterinary Medical Association.

The committee is composed of one delegate from each county brucellosis committee and one representative from the Pennsylvania State Veterinary Medical Association. The State committee meets once yearly to review the progress of the program and pass on resolutions presented from district meetings. An executive committee, appointed by the State committee, meets with the Pennsylvania Department of Agriculture to make recommendations for a better program.

Some large milk markets affecting Pennsylvania dairymen have already passed ordinances that require all dairymen shipping milk to these markets to test their herds for brucellosis. Many more markets are expected to follow suit.

\section{. . not in any newspapers . .}

"It is heartening to know that all over the world there are very large numbers of people quietly working away at their jobs, getting their feet wet and their hands dirty, coping with heat and cold and wet and dryness and fatigue and frustration, and sometimes discouragement, but they do carry on. Nobody knows about them; their names are not in any newspapers; they are hardly ever pictured by anyone. But these are the people who are making the real contacts between nations and between races and groups, and these are the people who need your support."

-Dr. Brock Chisholm, former Director-General, World Health Organization 